\title{
NUTZUNG REGENERATIVER ENERGIE ALS TRÄGER MECHANISCHER LEISTUNG IN DER SAVANNENREGION WESTAFRIKAS
}

\author{
René von Lipinski, Jörg Reiff-Stephan
}

\section{Zusammenfassung}

Ein Großteil des afrikanischen Kontinents ist noch immer von Armut betroffen. Im besonderen Maße ist dieser Zustand prägend für die westafrikanische Republik Togo. Kennzeichnend für die Region ist, dass sie über ein sehr schlecht ausgebautes Energienetz verfügt. Dieses hindert die Entwicklungsbedingungen sowohl im wirtschaftlichen als auch bildungspolitischen Bereich. Um die Weiterentwicklung des Landes zu fördern, hat sich die Technische Hochschule Wildau zum Ziel gestellt, Projektarbeiten im Bereich der Nutzbarmachung regenerativer Energien zu unterstützen und so einen Beitrag zur „Hilfe durch Selbsthilfe“ zu leisten. Im folgenden Artikel wird eine Analyse der Savannenregion hinsichtlich der klimatischen Bedingungen aufgezeigt. Die Analyseergebnisse dienen als Basis für die Ableitung möglicher regenerativer Energieträger und Konzepte zu deren dezentraler Nutzung. Am Fallbeispiel einer Lehmsteinpresse wird gezeigt, inwieweit der für die Region optimale regenerative Energieträger sinnvoll in mechanische Leistung überführt werden kann. Eine Berechnungsgrundlage zur Auslegung entsprechender Leistungsbedarfe wird abgeleitet. Hiermit wird ein Beitrag zum nachhaltigen Verbessern der Lebensbedingungen in Westafrika geleistet.

\begin{abstract}
A large part of the African continent suffers under the burden of poverty. The West African Republic of Togo suffers particularly heavily. Significant for the region is the very poorly developed energy grid. The lack of dependable power retards development in both economic and educational institutions. To promote development in the country, the University of Applied Sciences Wildau aims to support project work in the field of harnessing renewable energy, with the goal of "helping through self-help." In the following article an analysis of the savanna region's climatic conditions is performed to identify potential renewable energy sources. The main topic of the paper is to identify the optimal decentralized, renewable energy system for the region. Following that, the possibility of meaningful utilization for conversion into mechanical power is demonstrated in a case study of a brick making machine. A calculation basis for the design of appropriate performance requirements is derived. With this, a sustainable improvement to the living conditions in West Africa can be made.
\end{abstract}

\section{EINFÜHRUNG}

Ein Großteil des afrikanischen Kontinents ist noch immer von Armut betroffen. Im besonderen Maße ist dieser Zustand prägend für die westafrikanische Republik Togo, welche zu den ärmsten Ländern der Welt zählt [Kohnert 2006; Pohl und Kappel 2012]. Nach dem Bruttoinlandsprodukt mit einer Höhe von 3,8 Milliarden US\$ in 2012 (Deutschland: 3,4 Billionen US\$) beurteilt, liegt das Land an Stelle 151 der weltweiten Rangfolge [Info.org 2013]. Gerade in der nördlichen Savannenregion verfügt die Bevölkerung nur über begrenzte finanzielle Mittel. Das durchschnittliche Einkommen liegt in etwa zwischen 30 und 120 Euro pro Jahr. Die Armutsur- sachen sind vielschichtig und teilweise noch in der kolonialen Vergangenheit begründet. Die Infrastruktur des Landes ist gleichermaßen schlecht ausgeprägt. Die Nord-Südtangente ist durch eine einfache Straßenverbindung ausgeführt. Die Erreichbarkeit der angrenzenden Gebiete ist stark abhängig von der Bodenbeschaffenheit und dem Erosionsgrad infolge der klimatischen Bedingungen. Mit diesem Misstand geht einher, dass eine zentrale Energieversorgung und -verteilung nur bis Landesmitte sichergestellt ist. Aufgrund der schwachen Infrastruktur der nördlichen Regionen besteht in abgelegenen Ansiedlungen kein Zugang zu elektrischem Strom, fließendem Wasser oder Kanalisation. Gleichermaßen sind diese Gebiete jedoch stark auf den Nutzen elektrischer Energieträger angewiesen: beispielsweise zum Betreiben von Pumpsystemen oder ähnlichen mechanischen Hilfselementen sowie Beleuchtungssystemen [Löfken 2011]. Das Fehlen dieser grundsätzlichen Möglichkeiten hemmt die Entwicklung des Landes, wobei dies vor allem für das Bildungssystem der Region zutrifft. Ohne elektrische Energie kann der Unterricht nur bei Tageslicht erfolgen. In den Abendstunden können die Schüler nur im begrenzten Maße ihre Hausaufgaben bearbeiten, da zumeist Öllampen und Kerzen die einzigen Lichtquellen darstellen. Gleiches gilt für die Unterrichtsvorbereitung der Lehrer. Des Weiteren kann unter den gegebenen Umständen keine Schulspeisung gewährleistet wer- 
den. Der Mangel an Energie erschwert aber nicht nur den Betrieb des Schulsystems, sondern auch dessen Ausbau. Für die Region Nordtogo ist eine akute Unterversorgung an Schulplätzen zu verzeichnen. Die durchschnittliche Klassenstärke liegt bei etwa 70 Kindern [IT-Village 2013]. In Abb. 1 ist dieser Umstand veranschaulicht.

Die Gelder für die Schaffung neuer Kapazitäten sind knapp bemessen. Trotzdem ist auf entsprechenden Baustellen die kostenintensive Nutzung von Dieselgeneratoren die einzige Art der Stromerzeugung.

Um aktiv eine Änderung dieser Umstände zu bewirken, engagiert sich der in Greifswald ansässige Verein für „Deutsch Afrikanische Zusammenarbeit e.V.“ (DAZ e.V.) unterer anderem beim Bau neuer Schulen bzw. bei der Erweiterung und der energetischen Modernisierung bestehender Lehreinrichtungen. Partner vor Ort ist hierbei das IT Village in Dapaong. Durch die Technische Hochschule Wildau [FH] erfahren beide Organisationen Unterstützung bei der Entwicklung solartechnischer Komponenten und Systeme. Wesentliches Ziel der partnerschaftlichen Unterstützung ist es, der Bevölkerung im nördlichen Togo Wege für die Nutzung des hohen Potenzials regenerativer Energiesysteme aufzuzeigen. Hierbei stehen vor allem dezentrale Lösungen mit Leistungen im einstelligen Kilowattbereich im Fokus. Im Rahmen dieser Arbeit werden zunächst die Anforderungen seitens der klimatischen Bedingungen sowie an einen möglichen Träger regenerativer Energie analysiert. Systemanforderungen und Analyseergebnisse fließen dann in ein strukturiertes Bewertungsverfahren ein, um daraus den geeignetsten Energieträger südlich der Sahara abzuleiten. Der Nutzen wird anhand des Fallbeispiels „Lehmsteinpresse für die Herstellung von Grundmaterialien zum Schulbau“ erörtert.

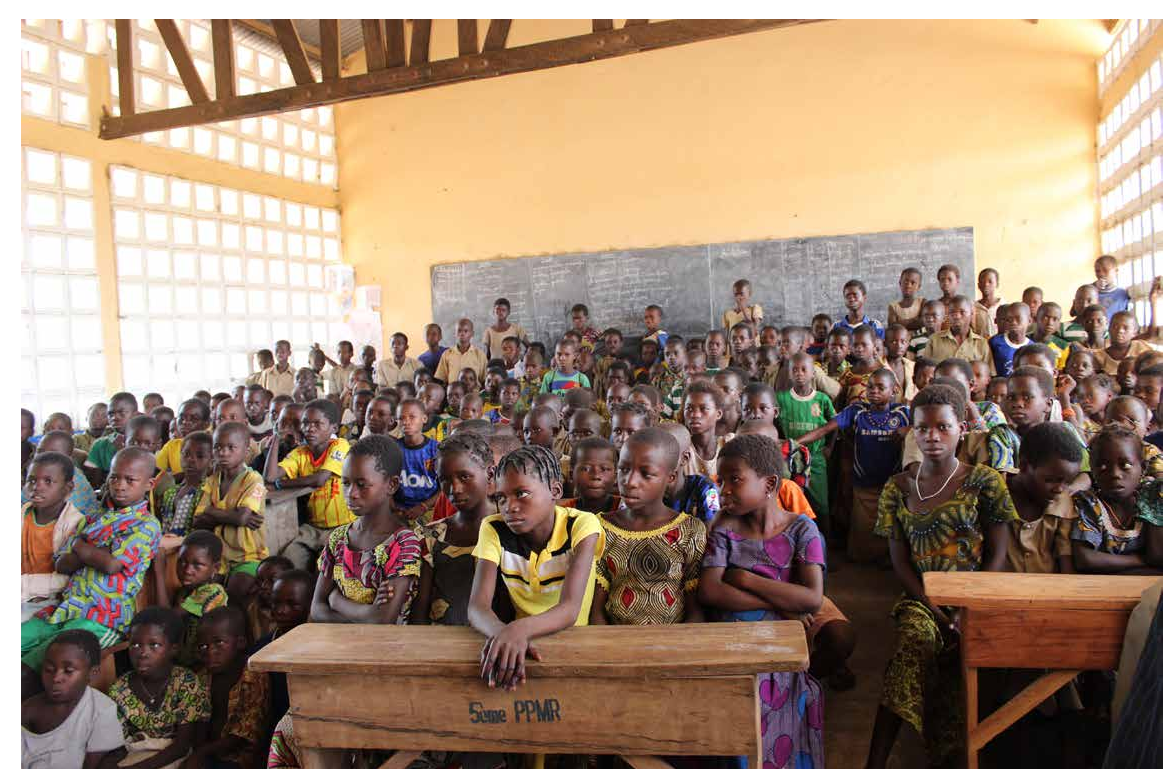

Abb. 1) Nordtogoische Unterrichtsbedingungen am Bespiel einer Schulklasse in Kourdjoak .

\section{ANALYSE DER KLIMATISCHEN UND TECHNISCHEN RAHMENBEDINGUNGEN}

Aufgrund der Lage Togos zwischen dem 6. und 12. Breitengrad sind im Jahresdurchschnitt täglich in etwa 6,5 Sonnenstunden mit einer vergleichsweise geringen Schwankungsbreite zu verzeichnen (Abb. 2).

Das Klima Togos ist ganzjährig tropisch-feucht. Die südlichen Regionen werden hierbei durch den Atlantik beeinflusst, was zu einer ganzjährig hohen Luftfeuchtigkeit von etwa 80 \% führt. Im Süden betragen die Temperaturen im Durchschnittlich $27{ }^{\circ} \mathrm{C}$ und es kommt jährlich zu zwei Regenzeiten zwischen den Monaten April und Juni sowie September und November. Der Norden des Landes ist eher durch trockenes, savannenartiges Klima geprägt. Die Temperaturen liegen im Durchschnitt bei $30^{\circ} \mathrm{C}$ mit einer Regenzeit zwischen Mai und Oktober.

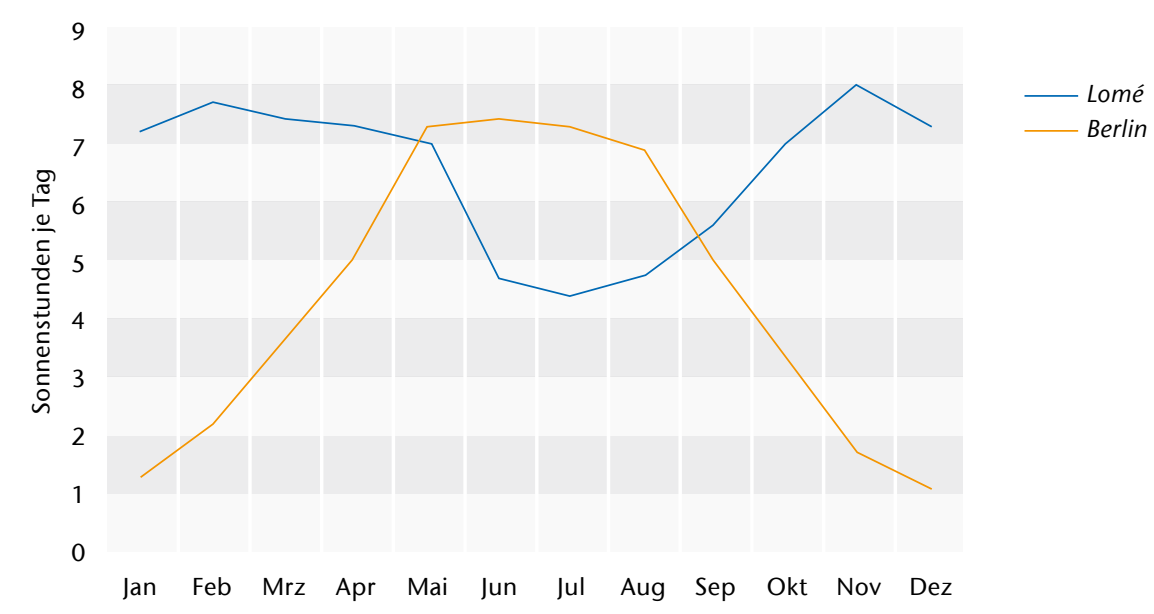

Abb. 2) Sonnenscheindauer im Jahresverlauf für Lomé (Hauptstadt der Republik Togo) im Vergleich zu Berlin [Klima.org 2013]. 
Die Entwicklung einer transportablen Anlage zur dezentralen Energieumwandlung muss insbesondere eine einfache Bedienbarkeit sowie einen geringen Wartungsaufwand berücksichtigen, da Mangel an qualifiziertem Personal besteht. Im Vielvölkerstaat Togo mit 42 Ethnien und 39 Sprachen, ohne dass alle Bevölkerungsteile die Amtssprache Französisch beherrschen, müssen die Bedienungsanforderungen die Wahrscheinlichkeit von Fehlbedienungen mindern. Für den Fall von Schäden am System ist ein geringer Instandsetzungsaufwand von Vorteil, da neben dem Fachkräftemangel auch mit Schwierigkeiten bei der Ersatzteilbeschaffung zu rechnen ist. Insbesondere ist eine geringe Störanfälligkeit anzustreben, wobei den extremen klimatischen Bedingungen Rechnung getragen werden muss. Bei der Umsetzung einer entsprechenden Lösung, sollte somit nicht an die Grenzen des technisch Machbaren gegangen werden. Im Sinne von Hilfe zur Selbsthilfe soll die Bevölkerung motiviert werden, derartige Systeme zu kopieren, um diese dann vielfältig anzuwenden. Hierfür sind vor allem ein geringer Herstellungsaufwand sowie geringe Herstellungskosten eine Voraussetzung. Üblicherweise ist die Nutzung von Dieselgeneratoren die bevorzugte Weise der autarken Stromerzeugung. Diese weisen zwar relativ geringe Anschaffungskosten auf, sind jedoch während des Betriebs kostspielig. Gründe hierfür sind vor allem der hohe Wartungsaufwand sowie die Kraftstoffkosten. Eine praktikable Alternative zum Dieselgenerator muss demnach durch niedrige Betriebskosten, eine hohe Energieverfügbarkeit und ein möglichst geringes $\mathrm{Maß}$ an Umweltbeeinträchtigungen überzeugen.

Der Norden Togos verfügt über kein nennenswertes Stromnetz. Für die ländlichen Regionen des Landes ist somit die dezentrale und autarke Energieversorgung von besonderer Bedeutung.

\section{ANALYSE POTENZIELLER ENERGIETRÄGER}

Aus dem Spektrum der regenerativen Energieträger bzw. Wandlungsprozesse haben folgende Technologien Anwendungspotenzial in Nordtogo: Windkraftanlagen, Photovoltaiktechnologie, solarthermische Kraftwerke und die Nutzung von Biomassenenergieträgern. Im Folgenden soll genauer auf die jeweiligen Technologien eingegangen werden.

\section{WINDENERGIE}

Bei der Windkraftnutzung ist darauf zu achten, dass nicht mehr als etwa $60 \%$ der im Wind enthaltenen Leistung entzogen werden kann. Dies ist auf den Betz'schen Leistungsbeiwert von $C P, B e t z=0,593$ zurückzuführen, welcher bei einem idealen Geschwindigkeitsverhältnis $\zeta$ id $=$ v2 $/ \mathrm{v} 1=1 / 3$ erreicht wird. Der Wirkungsgrad für Windkraftanlagen wird demnach aus der genutzten Windleistung PN und nutzbaren Windleistung Pid berechnet [Quaschning 2010]:

$$
\eta=\frac{P_{N}}{P_{i d}}=\frac{P_{N}}{P_{0} \cdot c_{P, B e t z}}=\frac{c_{P} w}{c_{P, B e t z}}
$$

Für Anwendungen im unteren Leistungsbereich werden Kleinwindkraftanlagen genutzt. Wie bei großen Windparks sind auch hier Auftriebsläufer mit horizontaler Rotorachse dominierend. Dies liegt vor allem am schlechteren Wirkungsgrad vertikaler Windkraftanlagen. Deren Vorteil ist jedoch die von der Windrichtung unabhängige Arbeitsweise. Ein Nachteil aller Kleinwindkraftanlagen ist vor allem die eingeschränkte Arbeitshöhe der Rotoren, wodurch deutlich geringere Windgeschwindigkeiten für den Leistungsentzug resultieren. Dieser Nachteil wird bei der Betrachtung von Abb. 3 deutlich. Diese zeigt die durchschnittlichen Windgeschwindigkeiten in verschiedenen Höhen für den Standort Dapaong unter Berücksichtigung des Rauigkeitsprofils einer Umgebung mit savannenartiger Vegetation.

Die Windgeschwindigkeiten in $10 \mathrm{~m}$ Höhe sind sehr gering. Somit wären große Rotordurchmesser und hohe Masten erforderlich, was die Errichtung von Anlagen zur nennenswerten Stromerzeugung erschwert und deren Kosten erhöht. Als Ergänzung zu anderen Energieträgern hat die Nutzung von Kleinwindkraftanlagen jedoch ihre Daseinsberechtigung.

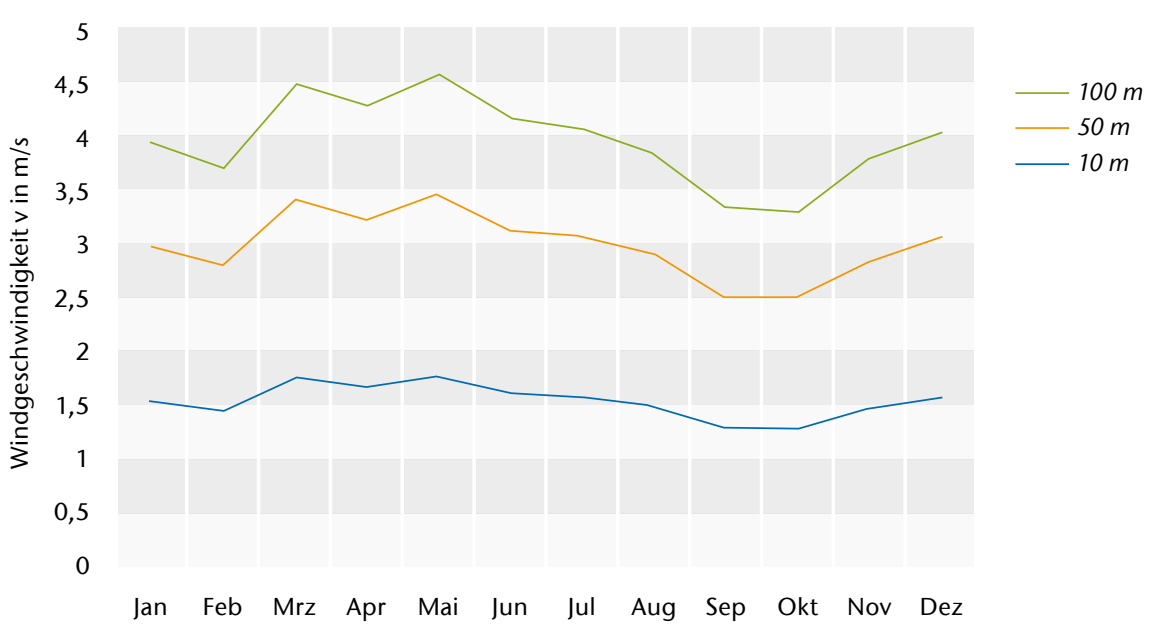

Abb. 3) Höhenbezogene Windgeschwindigkeiten am Standort Dapaong im Jahresdurchschnitt zwischen 1983 und 1993 [nasa.gov 2013]. 


\section{NUTZUNG VON SONNENENERGIE}

Verglichen mit den Ländern Mitteleuropas herrschen in Togo gute Bedingungen für die Nutzung der Photovoltaiktechnologie, da ganzjährig ein hohes Angebot an Strahlungsenergie vorliegt (Abb. 4).

Für die Photovoltaiktechnologie ergibt sich dadurch eine Vielzahl von Anwendungsmöglichkeiten in der Form von netzunabhängigen Insellösungen. Bei der Auslegung derartiger Anlagen muss jedoch beachtet werden, dass die extremen klimatischen Bedingungen die PV-Module sowie die elektrochemischen Speicherelemente stark beanspruchen. dies einen konstruktiven und steuerungstechnischen Mehraufwand. Aus diesem Grund rentieren sich derartige Anlagen nur an Standorten mit hohem Direktstrahlungsanteil, was für Togo nur begrenzt gegeben ist. Gegen den Einsatz von Dish-Stirling-Anlagen im Norden Togos sprechen jedoch vor allem der nachführungsbedingte Steuerungsaufwand sowie die hohen Investitionskosten.

\section{BIOMASSE}

Betrachtet man das Verhältnis der für die Biomassenproduktion benötigten Sonnenenergie und der letztlich in der Biomasse gespeicherten Energie, so ist ein sehr geringer Wirkungsgrad fest-

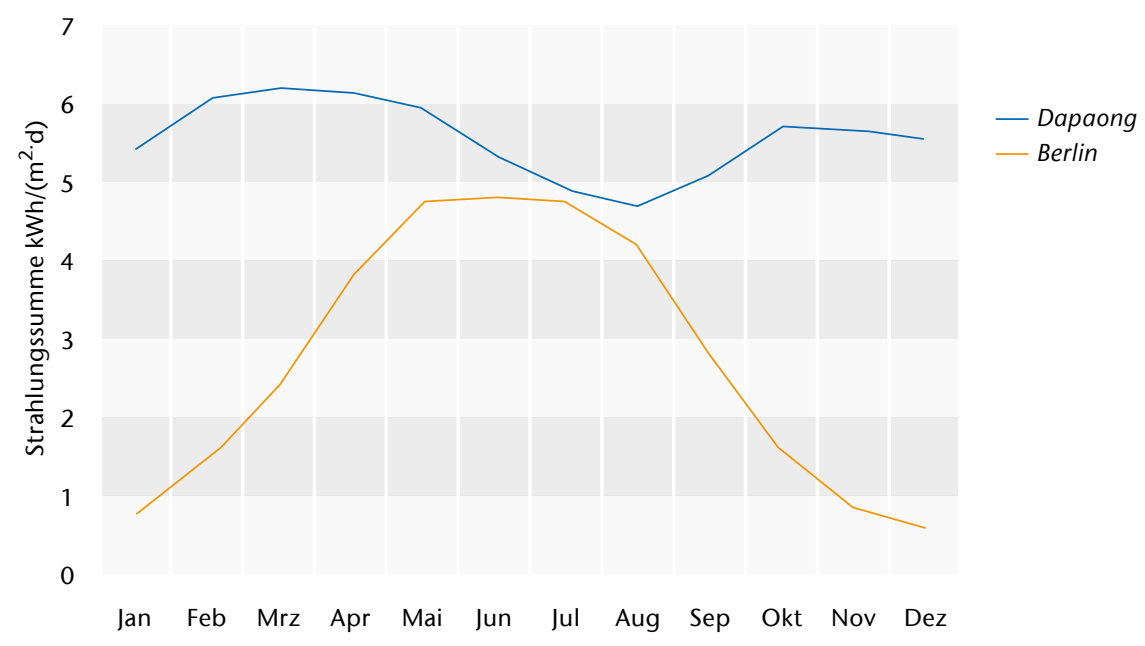

Abb. 4) Strahlungssumme auf eine horizontale Fäche am Standort Dapaong im Jahresdurchschnitt zwischen 1983 und 2005 [nasa.gov 2013].

Solarthermische Kraftwerke nutzen die Strahlungsenergie der Sonne und wandeln diese in Wärme und anschließend in elektrische Energie um. Für Anwendungen im unteren Leistungsspektrum bieten Dish-Stirling-Anlagen ein nennenswertes Potential. Diese arbeiten nach dem CSP-Prinzip (Concentrated Solar Power). Das Sonnenlicht wird mit Hilfe eines schalenförmigen Kollektors punktförmig konzentriert und die hieraus resultierende thermische Energie einem Stirling-Motor zugeführt. Um das Sonnenlicht beständig im Brennpunkt zu konzentrieren, muss der Kollektor kontinuierlich, zweiachsig nachgeführt werden [Laing et al. 2002]. Im Vergleich zur Photovoltaiktechnologie, welche auch die diffuse Strahlung nutzen kann, bedeutet zustellen. Dem steht jedoch eine gute Speicherfähigkeit als entscheidender Vorteil gegenüber. Die Nutzung von Biomasse zur Energieumwandlung kann durch eine Vielzahl von festen, flüssigen oder gasförmigen Energieträgern erfolgen. Durch direkte thermische Umwandlung oder direkte thermochemische Umwandlung wird die im Biomassenbrennstoff gespeicherte Energie freigesetzt [Kaltschmitt et al. 2001]. Die thermische Energie kann dann für verschiedene Zwecke genutzt oder durch entsprechende Verfahren in elektrische oder mechanische Energie umgewandelt werden. Die Erzeugung von Biomassenbrennstoffen erfolgt durch Aufbereitungsprozesse aus Abfällen, Reststoffen und Energiepflanzen [Quaschning
2010]. Der gezielte Anbau von Energiepflanzen ist jedoch nicht nur in Entwick-lungsländern wie Togo kritisch zu betrachten. Verglichen mit der Photovoltaiktechnologie benötigt die Energieumwandlung mit Biomasse für die gleiche Menge an elektrischer Ener-gie das 50-fache an Fläche [Mertens 2011]. Aufgrund des begrenzten Angebots an fruchtbaren Ackerflächen ist der Anbau von Nahrungsmitteln, der Energiepflanzenproduktion vorzuziehen.

Die energetische Verwertung von Abfällen und Reststoffen ist in diesen Regionen aber durchaus sinnvoll, da so auch die Probleme des Müllentsorgungssystems gemindert werden können. Gegenwärtig werden die Abfälle auf dafür vorgesehenen Plätzen gesammelt und in regelmäßigen Abständen verbrannt. Durch entsprechende Müllverbrennungsanlagen könnte die thermische Energie zur stationären Stromerzeugung genutzt werden. Für nicht ortgebundene Anwendungen in abgelegenen Gegenden ist dagegen die Auslegung der Anlagen schwierig. Wird der Energieträger nicht mitgeführt, ist mit starken qualitativen und quantitativen Schwankungen bei der Brennstoffversorgung zu rechnen. Des Weiteren erzeugt die Umwandlung von Biomasse in elektrische Energie einen hohen Anteil Prozesswärme. Da aber in Gebieten mit heißem Klima nur wenig Bedarf für eine KraftWärme-Kopplung (KWK) besteht, muss dieser Energieanteil durch eine Kraft-Wärme-Kälte-Kopplung (KWKK) genutzt werden. Demnach ist bei der Planung auf ein sinnvolles Nutzungskonzept zu achten.

\section{GEGENÜBERSTELLUNG}

Die Auswahl eines geeigneten Energieträgers für die nordtogoische Savannenregion erfolgt anhand einer Nutzwertanalyse (NWA) nach VDI 2225 (Tab. 1). Als wesentliche Kriterien der NWA wurden technologiebezogene und wirtschaftliche Parameter hinzugezogen. Starke Unterschiede treten bei der Betrachtung der Verfügbarkeit des Primärenergieträgers am Standort „Nordtogo“ sowie in der sozialen und Umweltverträglichkeit der Technologie auf. Es ist zu beobachten, 
dass insbesondere energiepflanzenbasierte Biomassesysteme elementar entgegen den humanitären Möglichkeiten agieren. Sowohl der Anbau von Biomasse als auch der Verbrauch von Abfallprodukten und Reststoffen kann zur mobilen Stromerzeugung nicht sinnvoll eingesetzt werden. Hinsichtlich der zu erwartenden Investitionskosten muss angeführt werden, dass Erstinstallationen weitestgehend über ausländische Hilfsorganisationen abgedeckt werden. Dennoch sollte mit der Korrelation eines Promotions- bzw. Entwicklungspotenzials der Anlage die Vervielfältigung der Technologie Berücksichtigung finden. Abschließend ist es wesentlich, dass die auszuwählende Technologie einen möglichst geringen Betriebs- und Wartungsaufwand hat und in der Nutzung robust agiert. Eine tägliche Einflussnahme durch zu schulendes Personal sollte möglichst nicht erforderlich sein.

Es ist zu erkennen, dass die Photovoltaiktechnologie mit einem Erfüllungsgrad von $75 \%$ der Ideallösung am nächsten kommt. Sie verfügt zwar im Vergleich mit den Windgeneratoren über einen schlechteren Wirkungsgrad, jedoch ist aufgrund der nied- rigen Windgeschwindigkeiten am Standort nicht mit einem hohen Ertrag zu rechnen. Des Weiteren zeigt die Betrachtung der Stromgestehungskosten, dass die Energieumwandlung mit PV-Anlagen, im Vergleich zum Dieselgenerator, weitaus günstiger zu bewerkstelligen ist. Somit kann die Photovoltaiktechnologie unter den gegeben Umständen als am besten geeigneter Energieträger für den Norden Togos angesehen werden.

\section{FALLSTUDIE LEHMSTEINPRESSE}

Eine Nutzungsmöglichkeit regenerativer Energie bietet der Alternativantrieb einer manuellen Lehmsteinpresse. Diese wird, wie anfangs erwähnt, auf Initiative des IT-Village und des DAZ e.V. für die Erweiterung und den Neubau von Schulgebäuden in Nordtogo genutzt. Mit der Lehmsteinpresse vom Typ Terstaram (Abb. 5) des belgischen Herstellers ApproTechno werden pro Stunde durchschnittlich 60 Lehmsteine gefertigt. Bei einem zehnstündigen Arbeitstag liegt die Tagesproduktion bei 600 Lehmsteinen.

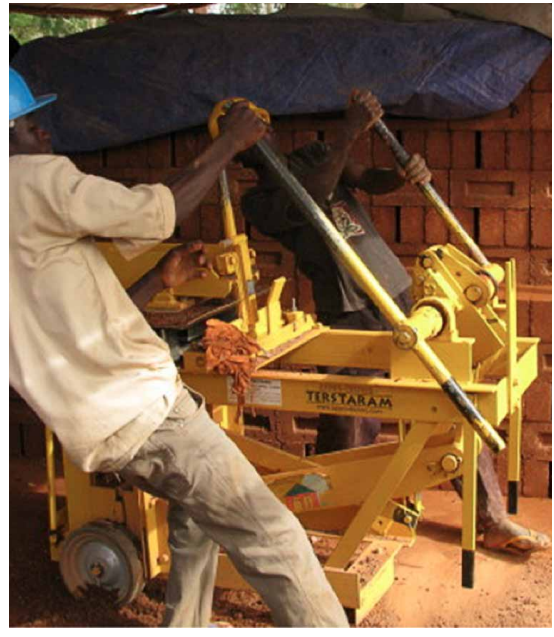

Abb. 5) Togoische Bauarbeiter bei der Bedienung einer manuellen Lehmsteinpresse vom Typ ApproTechno Terstaram [DAZ e.V. 2013]

\begin{tabular}{|c|c|c|c|c|c|c|c|c|c|c|c|}
\hline \multicolumn{2}{|c|}{ Bewertungskriterien } & \multicolumn{2}{|c|}{ Windenergie } & \multicolumn{2}{|c|}{ Photovoltaik } & \multicolumn{2}{|c|}{$\mathrm{STK}^{* * *}$} & \multicolumn{2}{|c|}{ Biomasse } & \multicolumn{2}{|c|}{$\begin{array}{c}\text { Diesel } \\
\text {-generator }\end{array}$} \\
\hline technologisch & $G^{*}$ & $\mathrm{~B}^{* *}$ & $\mathrm{G} \cdot \mathrm{B}$ & - & - & - & - & - & - & - & - \\
\hline Energieverfügbarkeit & $20 \%$ & 3 & 0,6 & 8 & 1,6 & 7 & 1,4 & 2 & 0,4 & 1 & 0,2 \\
\hline Wirkungsgrad (Primär) & $15 \%$ & 8 & 1,2 & 4 & 0,6 & 5 & 0,75 & 2 & 0,3 & 2 & 0,3 \\
\hline Umweltverträglichkeit & $10 \%$ & 9 & 0,9 & 8 & 0,8 & 8 & 0,8 & 3 & 0,3 & 1 & 0,1 \\
\hline Soziale Verträglichkeit & $20 \%$ & 9 & 1,8 & 8 & 1,6 & 7 & 1,4 & 1 & 0,2 & 1 & 0,2 \\
\hline Entwicklungspotenzial** & $10 \%$ & 9 & 0,9 & 9 & 0,9 & 4 & 0,4 & 2 & 0,2 & 1 & 0,1 \\
\hline wirtschaftlich & - & - & - & - & - & - & - & - & - & - & - \\
\hline Investitionskosten & $5 \%$ & 7 & 0,35 & 5 & 0,25 & 1 & 0,05 & 8 & 0,4 & 8 & 0,4 \\
\hline Stromgestehungskosten & $5 \%$ & 8 & 0,4 & 7 & 0,35 & 5 & 0,25 & 4 & 0,2 & 2 & 0,1 \\
\hline Wartungskosten & $15 \%$ & 7 & 1,05 & 9 & 1,35 & 2 & 0,3 & 5 & 0,75 & 3 & 0,45 \\
\hline$\Sigma$ & - & - & 7,2 & - & 7,5 & - & 5,4 & - & 2,8 & - & 1,9 \\
\hline Erfüllungsgrad & $100 \%$ & - & $72 \%$ & - & $75 \%$ & - & $54 \%$ & - & $28 \%$ & - & $19 \%$ \\
\hline
\end{tabular}


Für die Produktion der besagten Menge an Lehmsteinen müssen momentan in etwa 660 Wh körperlicher Arbeit aufgebracht werden. Diese Aussage beruft sich auf folgende überschlägige Berechnung. Es wird davon ausgegangen, dass während des Pressvorgangs zwei Arbeiter mit einer Kraft F von jeweils $400 \mathrm{~N}$ die Hebel der Presse bedienen. Die Hebel haben eine Länge I von 0,7 m. Aus Gleichung (4) ergibt sich ein resultierendes Drehmoment $M=560 \mathrm{Nm}$ (Abb. 6).

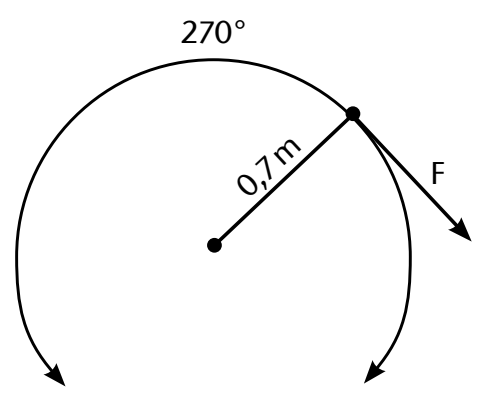

(2) $M=F \cdot l=2 \cdot 400 \mathrm{~N} \cdot 0,7 \mathrm{~m}=560 \mathrm{Nm}$

Abb. 6) Modell Pressenantrieb

Die Drehbewegung erfolgt in einem Winkel von $270^{\circ}$ und einer Drehzahl von $\mathrm{n}$ $=10$ 1/min. Werden diese Parameter in Gleichung (5) übertragen, so ergibt sich eine Leistung von $\mathrm{P}=587 \mathrm{~W}$.

Bei 600 Pressvorgängen pro Tag mit einer Dauer von jeweils $t=4,5 \mathrm{~s}$ und der $z u-$ vor bestimmten Leistung ergibt sich für die benötigte Energie ein Zusammenhang nach Gleichung (6) zu:

(3) $P=M \cdot \omega=M \cdot 2 \cdot \pi \cdot n=560 \mathrm{Nm} \cdot 2 \cdot \pi \cdot 0,1671 / \mathrm{s}=587 \mathrm{~W}$

Für die Pressvorgänge ist somit eine Energie von EP $=0,44$ kWh nötig. Bei der Annahme, dass für das Lösen der Presse nur die Hälfte der Kraft nötig ist, ergibt sich bei analoger Vorgehensweise ein Energiebedarf von $\mathrm{EL}=0,22 \mathrm{kWh}$.

-

$E=P \cdot t=587 \mathrm{~W} \cdot 4,5 \mathrm{~s} \cdot 600=0,587 \mathrm{~kW} \cdot 0,00125 \mathrm{~h} \cdot 600=0,44 \mathrm{kWh}$

In der Summe ist für die Produktion von 600 Lehmsteinen eine Gesamtenergie von $\mathrm{EG}=0,66 \mathrm{kWh}$ nötig. Soll das durch die Arbeiter bewirkte Drehmoment nun maschinell erzeugt werden, so müssen zusätzlich die Wirkungsgrade für Motor und Getriebe berücksichtig werden. Für das System Pressenantrieb ist demnach eine elektrische Eingangsleistung von Pel, $\mathrm{p}=814 \mathrm{~W}$ bzw. Pel, I $=407 \mathrm{~W}$. Für den maschinellen Antrieb der Presse wird somit eine Energie von Eel $=0,916 \mathrm{kWh}$ benötigt (Gleichung (7):

(5) $E_{\mathrm{el}}=P_{\mathrm{el}, \mathrm{p}} \cdot t_{p} \cdot 600+P_{\mathrm{el}, 1} \cdot t_{1} \cdot 600=(0,814 k W+0,407 \mathrm{~kW}) \cdot 0,00125 h \cdot 600=0,916 \mathrm{kWh}$

Die Bereitstellung dieser Energiemenge soll durch ein Inselsystem auf Basis der Photovoltaiktechnologie gewährleistet werden. Zentrale Systemkomponenten sind hierbei PV-Generator und Akkumulator. Für die Dimensionierung dieser Komponenten mussten die Modulneigung und die täglich zur Verfügung stehenden Strahlungssummen geklärt werden. Es zeigte sich jedoch, dass unter den gegebenen Umständen auf eine größere Neigung der Module verzichtet werden kann. Für das ungehinderte Ablaufen von Wasser bei Niederschlägen oder der Modulreinigung ist ein kleiner Modulanstellwinkel von 5 bis $15 \mathrm{Grad}$ ausreichend. Die erste überschlägige Berechnung der benötigten Leistung des PV-Generators erfolgt nach Gleichung (8) mit Parametern nach Tab. 2 [Mertens 2011].

6 $P_{\mathrm{PV}}=\frac{\mathrm{W}}{N_{\text {Sonne }} \cdot K_{\text {Schräg }} \cdot K_{\mathrm{Temp}} \cdot V_{\text {Leitung }} \cdot V_{\text {Umwandlung }} \cdot V_{\text {Anpassung }}}=475 \mathrm{Wp}$
Für den PV-Generator ist somit eine Modulleistung von insgesamt PPV = $475 \mathrm{Wp}$ vorzusehen. Zur überschlägigen Bestimmung der Akkukapazität $\mathrm{CN}$ kommt Gleichung (9) zur Anwendung. Die entsprechenden Parameter sind Tab. 3 zu entnehmen [Mertens 2011].

(7) $C_{\mathrm{N}}=\frac{W \cdot N_{\mathrm{A}}}{K_{E n t l} U_{\mathrm{N}}}=153 \mathrm{Ah}$

Die zuvor berechneten Werte für PPV und $\mathrm{CN}$ dienen als Orientierungsgrundlage, um eine Vorauswahl an verwendbaren PV-Modulen und Akkumulatoren zu treffen. Die exakte Dimensionierung des Systems erfolgt mit dem Auslegungsprogram PV*SOL Expert. In dessen Datenbank sind die Parameter der gängigsten PV-Module und Akkumulatoren hinterlegt. Mit Hilfe der Software können für die jeweils gewählte Komponentenkombination entsprechende Szenarien simuliert und verglichen werden. Je nach Zelltechnologie und Berechnungsparameter ist für die spätere Umsetzung des PV-Generators eine Fläche von 3,5 $-5 m^{2}$ zu erwarten.

Da sich der Standort der Energieversorgungseinheit entsprechend der jeweiligen Baustelle ändert, muss die Mobilität des Systems gewährleistet sein. Hierfür wird der PV-Generator auf einen Anhänger montiert (Abb. 7).

Die Straßenverhältnisse in den nordtogoischen Regionen können als schlecht bezeichnet werden. Um die Verwindungen im PV-Generator möglichst gering zu halten, ist für das Trägersystem ein einachsiger Anhänger vorgesehen. Zusätzlich werden anstelle weniger großer Module mehrere kleine und dadurch steifere Module verwendet. Zusätzlich müssen diese mit ausreichend Abstand zum Anhänger sowie zueinander montiert werden, um eine gute Hinterlüftung der Module zu ermöglichen. Da Wasser in der Region ein kostbares Gut ist, sind unterhalb des PV-Generators Rinnen vorgesehen. Auf diese Weise kann das zur Modulreinigung genutzte Wasser aufgefangen und als Brauchwasser genutzt werden. Für die Energiespeicherung sind konventionelle Solarbleiakkumulatoren vorgesehen. 


\begin{tabular}{|c|c|c|c|}
\hline Benennung & & Wert & Einheit \\
\hline Energiebedarf & W & 916 & Wh \\
\hline Sonnenvolllaststunden & Nsonne & 4,55 & $\mathrm{~h}$ \\
\hline Korrekturfaktor & Kschräg & 1 & - \\
\hline Korrekturfaktor & KTemp & 0,625 & - \\
\hline Leitungsverlustfaktor & VLeitung & 0,94 & - \\
\hline Umwandlungsverlustfaktor & Vumwandlung & 0,8 & - \\
\hline Anpassungsverlustfaktor & VAnpassung & 0,9 & - \\
\hline
\end{tabular}

Tab. 2) Technische Systemparameter I/II

\begin{tabular}{|c|c|c|c|}
\hline Benennung & & Wert & Einheit \\
\hline Energiebedarf & W & 916 & Wh \\
\hline Autonomietage & $\mathrm{NA}_{\mathrm{A}}$ & 2 & - \\
\hline Korrekturfaktor Entladeschwelle & $\mathrm{K}_{\mathrm{Entl}}$ & 0,5 & - \\
\hline Systemspannung & $\mathrm{UN}_{\mathrm{N}}$ & 24 & $\mathrm{~V}$ \\
\hline
\end{tabular}

Tab. 3) Technische Systemparameter II/III

Die Umwandlung und Übertragung der Energie zur Lehmsteinpresse erfolgt über einen Elektromotor und ein Getriebe. Die Einbindung dieser Komponenten in das bisherige System der Lehmsteinpresse erfolgt unter der Vorgabe, dass die Option zum manuellen Betrieb erhalten bleibt. Des Weitern ist das System so aufgebaut, dass einen Nutzung der PV-Anlage auch unabhängig von der Lehmsteinpresse für andere Zwecke erfolgen kann.

\section{AUSBLICK}

Die Verfügbarkeit elektrischer Energie ist eines der wesentlichen Grundelemente für wirtschaftliches Wachstum und humanitäre Lebensbedingungen. In einer der ärmsten Regionen Westafrikas - in der Savannenregion Nordtogos - ist der zentrale Ausbau von Stromnetzen nicht erfolgt. Dezentrale Lösungen auf Basis regenerativer Energieträgersysteme können hier AbhilfeschaffenunddieEntwicklungder Region vorantreiben. In der vorliegeden Arbeit wurden die verschiedenen, regenerativen Energieträgersysteme auf ihren Einsatz in der Savannenregion Westafrikas hin untersucht. Ausführend konnte das solartechnische Energieträgersystem im Einsatz

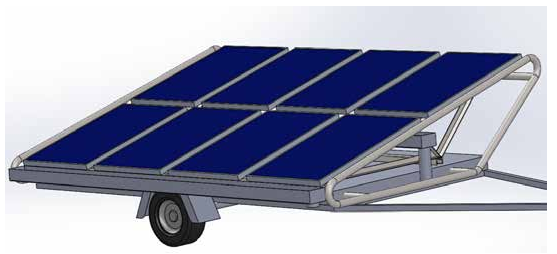

Abb. 7) CAD-Modell der mobilen Photovoltaikanlage

als Leistungslieferant im sekundären Wandlungsprozess zu mechanischer Leistung herangezogen werden. In der Folge ist ein Prototyp für eine Lehmsteinproduktionsmaschine konstruktiv ausgelegt und ein digitales Mock-up erarbeitet worden. Gemeinsam mit den Partnern in Togo wird der Prototyp nunmehr aufgebaut und exemplarisch im Feld getestet. Erfahrungen werden in Zusammenarbeit mit der Universität Lomé gesammelt und fließen in die Ausbildungsinhalte in Togo wie auch an der Technischen Hochschule Wildau [FH] mit ein. Hiermit wird ein wesentlicher Beitrag zur Nutzbarmachung der Technologie am Standort sowie zur Verbessern der Lebensbedingungen in Westafrika geleistet.

\section{LITERATUR}

DAZ e.V. (2013): www.daz-eu.de. online: http://www. daz-eu.de/index.php; Zugriff am 4. Dezember 2013

In-fo.org (2013): www.imf.org. online: http://www. imf.org/external/pubs/ft/weo/2013/02/weodata/index. aspx; Zugriff am 5. Dezember 2013

IT-Village (2013): www.it-village-togo.de. online: http:// www.it-village-togo.de/ohne\%20Schule\%20geht $\% 20$ es\%20nicht.html; Zugriff am 4. Dezember 2013

Kaltschmitt, M. und Hartmann, H. (2001): Energie aus Biomasse. Berlin: Springer Verlag.

Klima.org (2013): http://www.klima.org/togo/klimalome/. online: http://www.klima.org/togo/klima-lome/; Zugriff am 6. September 2013

Kohnert, D. (2006): Togo. In: Africa Yearbook 2005 - Politics, economy and society South of the Sahara. Melber/ Mehler/Walraven (eds.), Brill, Leiden \& Boston, in press

Laing, D.; Schiel, W. und Heller, P. (2002): Solare Kraftwerke. Berlin: ForschungsVer-bund Sonnenenergie

Löfken, J. O. (2011): Afrika entdeckt die Sonne. In: tr Technology Review (2011) 6, S. 26-32

Mertens, K. (2011): Photovoltaik - Lehrbuch zu Grundlagen, Technologie und Praxis. München: Carl Hanser Verlag.

nasa.gov (2013): https://eosweb.larc.nasa.gov/cgi-bin/ sse/sse.cgi/. online: https://eosweb.larc.nasa.gov/cgibin/sse/sse.cgi/; Zugriff am 23. August 2013

Pohl, B. und Kappel, R. (2012): Wie leistungsfähig sind die Ökonomien Afrikas. In: GIGAfokus (2012) 9, online: $<$ Www.giga-hamburg.de/giga-focus/africa> ISSN 18623603

Quaschning, V. (2009): Regenerative Energiesysteme. München: Carl Hanser Verlag

Quaschning, V. (2010): Erneuerbare Energie und Klimaschutz. München: Carl Hanser Verlag

\section{AUTOREN}

M. Eng. René von Lipinski

wissenschaftlicher Mitarbeiter Automatisierungstechnik Technische Hochschule Wildau [FH] von_lipinski@th-wildau.de

Prof. Hon.-Prof. Dr.-Ing. Jörg Reiff-Stephan

Professur Automatisierungstechnik/Produktionstechnik Technische Hochschule Wildau [FH] jrs@th-wildau.de 